\title{
Mitteilungen
}

\section{Hanse-Preise 2022}

\section{Hanse-Preis für Intensivmedizin 2022}

gestiftet

von der Fresenius-Stiftung Bad Homburg

Dotation: EUR 5000

Der Gewinner/die Gewinnerin des HansePreises für Intensivmedizin wird in einem Vortragswettbewerb ermittelt. Berücksichtigt werden 2021 publizierte Originalarbeiten (peer reviewed, experimentell oder klinisch, deutsch oder englisch) aus den Bereichen der Intensiv- oder Notfallmedizin.

Hanse-Pflegepreis für die fachpraktische Arbeit 2022

gestiftet von der B. Braun Melsungen AG

Dotation: EUR 2000

Hanse-Pflegepreis für die pflegewissenschaftliche Arbeit 2022

\section{gestiftet}

von der Vifor Pharma Deutschland GmbH

\section{Dotation: EUR 2000}

Die Gewinner der Hanse-Pflegepreise werden in einem Vortragswettbewerb ermittelt. Berücksichtigt werden veröffentlichte und unveröffentlichte Arbeiten aus den Bereichen der Intensiv- und Anästhesiepflege (auch Referate/Fach- und Jahresarbeiten im Rahmen der Fachweiterbildung).

Hanse-Förderpreis für Konzepte zur Verbesserung der intensivmedizinischen Versorgungsqualität 2022

gestiftet vom Wissenschaftlichen Verein zur Förderung der klinisch angewendeten Forschung in der Intensivmedizin e.V.

Dotation: EUR 3000

Der Förderpreis wird jährlich für innovative Projekte und Projektvorhaben vergeben, die auf eine nachhaltige Verbesserung der intensivmedizinischen und intensivpflegerischen Versorgungsqualität abzielen. Es werden vorzugsweise Projekte berücksichtigt, die anerkannte Instrumente des Qualitätsmanagements (PDCA-Zyklen) verwenden.

Hanse-Promotionspreis für klinische und experimentelle Forschung in der Intensivund Notfallmedizin 2022

gestiftet

von Achim Schulz-Lauterbach VMP GmbH

Dotation: je EUR 2000

Prämiert wird jeweils eine Promotionsarbeit aus den Bereichen Intensivmedizin oder
Notfallmedizin aus den Jahren 2020 und 2021 (Datum der Promotionsurkunde). Die Preisträger erhalten die Gelegenheit, ihre Arbeiten auf dem 32. Symposium 2022 vorzustellen.

\section{Hanse-Habilitationspreis 2022}

gestiftet

von der CytoSorbents Europe $\mathrm{GmbH}$

Dotation: EUR 10000

Ausgezeichnet werden bereits angenommene Habilitationsarbeiten aus den Jahren 2020 und 2021 (Datum der Habilitationsurkunde). Der Preisträger oder die Preisträgerin wird auf dem 32. Symposium 2022 vorgestellt und im Folgejahr als Referent oder Referentin zu einem Thema im Kontext der ausgezeichneten Habilitationsschrift eingeladen.

Einsendeschluss für alle Preise: 15. Dezember 2021 als PDF an: kontakt[at]wivim.org

Weitere Informationen finden Sie unter: wivim.org/preise

Auskünfte erteilt:

WIVIM e.V. Geschäftsstelle

Sandra Eylers

Sögestr. 48, DE-28195 Bremen

Tel.: +4930729820

E-Mail: Eylers.sandra[at]wivim.org

\section{Aktuelle Themen auf unserer Website www.saez.ch $\rightarrow$ Tour d'horizon
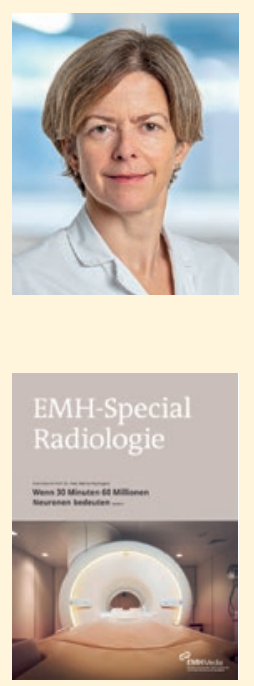 \\ Interview mit Prof. Dr. med. Maria Wertli, Präsidentin Qualitätskommission der Schweizerischen Gesellschaft für Allgemeine Innere Medizin (SGAIM) \\ Qualitätsindikatoren - Nutzen und Grenzen \\ Die Qualitätskommission der SGAIM präsentiert erstmals die neuen Qualitätsindikatoren für die stationäre Behandlung.

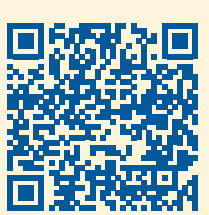 \\ Editorial von Sandra Ziegler, Geschäftsführerin EMH Schweizerischer Ärzteverlag \\ EMH-Special: eine neue Publikationsreihe \\ Spannende Berichte, wertvolle Inputs für den Praxisalltag und Erfahrungsaustausch - dies sind die wichtigsten Eckpunkte der neuen Publikationsreihe «EMH-Special».}

